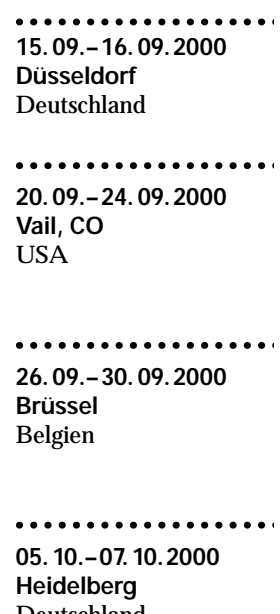

D eutschland

06. 10.-09. 10.2000

München

D eutschland

06. 10. 09. 10.2000

München

D eutschland

11 10.- 15. 10.2000
Traverse City, MI
U SA

USA

13. 10.- 17. 10.2000
Hamburg
Deutschland
$\ldots \ldots \ldots \ldots$
18. 10.-21. 10.2000
$\begin{aligned} & \text { Pich/Schladming } \\ & \text { Osterreich }\end{aligned}$

[..................

21. 10.- 25. 10.2000

Graz

Österreich

01. 11-04. 11.2000

Wilsede

D eutschland

\section{Onkologisches Symposium «Maligne Knochentumoren»}

Cytokines and Cancer: Regulation, Angiogenesis, and Clinical Applications

\section{2nd European Breast Cancer Conference}

10. J ahrestagung der Arbeitsgemeinschaft für Dermatologische Onkologie/Herbsttagung der EORTC-Melanomgruppe

Gemeinsamer J ahreskongress der Deutschen und Österreichischen Gesellschaft für Radioonkologie und Deutschen Gesellschaft für Medizinische Physik

Intraoperative Strahlentherapie als integraler Bestandteil eines multimodalen Therapiekonzeptes (im Rahmen des gemeinsamen Kongresses von DEGRO/ÖGRO/SASRO)

Angiogenesis and Cancer: From Basic Mechanisms to Therapeutic Application

\section{5th ESMO Anniversary and 25th ESMO Congress}

Austrotransplant 2000 - 14. J ahrestagung der Austrotransplant/ Österreichische Gesellschaft fürTransplantation, Transfusion und Genetik

Gemeinsame J ahrestagung der Deutschen und Österreichischen Gesellschaft für Hämatologie und Onkologie

Ausgewählte Kapitel zur Diagnostik und Therapie gastrointestinaler Tumoren
Auskunt: O rthopädische K linik der $\mathrm{H}$ einrich-H eine-U niversität, Frau $\mathrm{H}$ emmers M oorenstraße 5, D-40225 D üsseldorf Tel. +49 $211811-8314$, Fax -6693

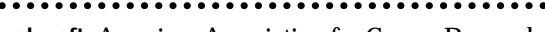
Public L edger B uilding, Suite 826

150 South Independence M all West

Philadelphia, PA 10106-3483, U SA

Tel. +1 440-9300, Fax -9165

Auskunft: $\mathrm{FECS}$ Conference U $\mathrm{U}$ nit

A venue $\mathrm{E}$. M ounier $83, \mathrm{~B}-1200 \mathrm{~B}$ russels

Tel. +32 2 77502-01, Fax -00

E-mail EBCC-2@fecs.be

www.fecs.be

Auskunft: Prof. Dr. Dirk Schadendorf

$\mathrm{Dr}$. U do H ofmann, K linische Kooperationseinheit

für D ermatoonkologie (DKFZ) an der Hautklinik des

U niversitätsklinikums $M$ annheim

Theodor-Kutzer-U fer 1, D -68135 M annheim

Tel. +49 621 383-2126, Fax -2163

E-mail d.schadendorf@dkfz-heidelberg.de

..................................

Auskunft: PD Dr.H.J. Feldmann

K linik für Strahlentherapie der TU M ünchen

$\mathrm{D}-81377 \mathrm{M}$ ünchen

Tel. +49 $89414045-12$, Fax - 45

E-mail degro2000@degro.org

www.degro.org

Auskunft: Kongress-Sekretariat / E SO -D

c/o ZeTuP, Frau H oevel

R orschacher Straße 150, CH -9006 St. G allen

Tel. +41 71 24-30032, Fax -56805

E-mail eso-d@sg.zetup.ch

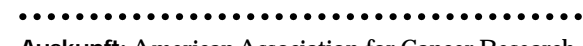

Auskunft: A merican A ssociation for $C$ ancer R esearch Public Ledger B uilding, Suite 826

150 South Independence M all West

Philadelphia, PA 10106-3483, U SA

Tel. +1 440-9300, Fax - 9165

www.aacr.org

Auskunft: ESM 0 Congress Secretariat

Via Soldino 22, CH-6900 L ugano

Tel. +41 91 950-0781, Fax -0782

E-mail esmo@dial.eunet.ch

Auskunft: K linische A bteilung für Pädiatrische

H ämatologie/O nkologie, LK H/U niversitätsklinikum

A uenbruggerplatz $30, \mathrm{~A}-8036 \mathrm{G}$ raz

Tel. +43 316 385-3485, Fax - 3450

E-mail ped.hemonk@kfunigraz.ac.at

www. austrotransplant2000.at

Auskunft: M edizinische A usstellungs- und

Werbegesellschaft

Freyung 6, A-1010 Wien

Tel. +43 153-6630, Fax -56016

E-mail maw@media.co.at

Auskunt: Prof. Dr. H.J. Illiger

Städt. K liniken, K linik für Innere M edizin II

z. H. Frau K lara Schlunk

D r.-E den-Straße 10, D -26133 O Idenburg

Tel. +49 4414 0326-11, Fax -54

\begin{tabular}{ll}
\hline KARGER & ○ 2000 S. Karger G mbH , Freiburg \\
Fax +49 761 4520714 & A ccessible online at: \\
$\begin{array}{l}\text { E-mail Information@K arger.de } \\
\text { www.karger.com }\end{array}$ & www.karger.com/journals/onk
\end{tabular}




\section{11-04. 11.2000}

Wilsede

D eutschland

\section{$0111-04.112000$}

Wilsed

D eutschland

\section{11-04. 112000 \\ Bad Nauheim}

D eutschland

\section{11.2000}

Heidelberg

D eutschland

[......................

07. 11- 10. 112000

Amsterdam

Niederlande

\section{11- 12.11 .2000}

Gravenbruch/

Frankfurt a. M.

D eutschland

10. $11-12.112000$

Ö sterreich

12. 11- 16. 112000
Eilat
I srael

\subsection{0 \\ Köln \\ D eutschland \\ 19. 11-20. 11.2000 \\ Wien \\ Ö sterreich}

24. 11-25. 112000

Dresden

D eutschland

Wien

Österreich

02.12.2000

D eutschland

0.000 .00 .00

21. 02.- 24. 02.2001

St. Gallen

Schweiz
Evidence-Based Medicine - Anleitung zur knitischen Analyse der Literatur am Beispiel gastrointestinalerTumoren

Neue Methoden und Forschungsansätze der Strahlentherapie

Gemeinsam gegen Krebs - Hessischer Krebskongress

TNM-Kongress 2000: Bedeutung desTNM-Systems für die Klinische Onkologie - Symposium des DSK-TNM

\section{1th NCI-EORTC-AACR Symposium on New Drugs in Cancer} Therapy

5. Darmstädter Live-Symposium für Operative Dematologie «Ästhetisch-operative Dematologie im neuen J ahrtausend»

16. Arbeitstagung der Österreichischen Gesellschaft für Psychoonkologie (ÖGPO)

New Targets for Cancer Prevention

Innovative Behandlungskonzepte und Techniken in der Radioonkologie

\section{Onkologie in Klinik und Praxis}

\section{Dresdner hämatologisch-onkologisches Gespräch}

\section{Methodik klinischer Prüfung in der Onkologie}

10. Symposium «Aktuelle Aspekte der Wundheilung», Schwerpunkt: Wundprobleme in der Onkologie

\section{Adjuvant Therapy of Primary Breast Cancer}

Auskunft: Prof. D r. H.J. Illiger

Städt. K liniken, K linik für Innere M edizin II

z. H. Frau K lara Schlunk

D r.-E den-Straße 10, D -26133 O Idenburg

Tel. +49 4414 0326-11, Fax -54

Auskuntt: Prof. D r. H.J. Illiger

Städt. K liniken, K linik für Innere M edizin II

z. H. Frau K lara Schlunk

D r.-E den-Straße 10, D -26133 O Idenburg

Tel. +49 441 40326-11, Fax -54

Auskunft: H ess. K rebskongress e.V.

Frau Richter

H einrich-H eine-Straße 44, D -35003 M arburg

Tel. +49642163324

Auskunft: Prof. D r. C. Wittekind

Institut für Pathologie der U niversität L eipzig

L iebigstraße 26, D-04103 L eipzig

Tel. +49 341 97150-00. Fax -09

Auskunft: $\vee \mathrm{U}$ Conference Service

D e B oelelaan 1105, N L-1081 H V A msterdam

Tel. +31 20 444-57 90, Fax -5825

E-mail vu_conference@dienst.vu.nl

www.nddo.nl/conferences

Auskunft: comed $\mathrm{GmbH}$, Kongressorganisation

R eichsgrafenstraße 10, D -79102 Freiburg

Tel. +49 761 79127-0, Fax -27

E-mail info@comed-kongresse.de

www.comed-kongresse.de

Auskunft: E rika Wasserrab

$R$ aiffeisenstraße $11, A-2326 L$ anzendorf

Tel. +43 22 35-47230, Fax -44816

E-mail oegpo@eunet.at

www.members.eunet.at/oegpo

Auskunft: A merican A ssociation for Cancer R esearch Public L edger B uilding, Suite 826

150 South Independence M all West Philadelphia

PA 10106-3483, U SA

Tel. +1 4 40-9300, Fax -9165

Auskunft: D r.-M ildred-Scheel-A kademie

J oseph-Stelzmann-Straße 9, D-50931 K öln

Tel. +49 $221944049-0$, Fax - 44

Auskunft: Kongress-Sekretariat / E SO -D

c/o ZeTuP, Frau H oevel

R orschacher Straße 150, CH -9006 St. G allen

Tel. +41 71 24-30032, Fax - 56805

E-mail eso-d@sg.zetup.ch

Auskunft: Med. K linik und Poliklinik I des

U niversitätsklinikums Carl Gustav Carus de

TU D resden, D r. H. G ünther

Fetscherstraße 74, D-01307 D resden

Tel. +49 351 458-2583, Fax -4373

E-mail guenther@oncocenter.de

Auskunft: $M$ ag. Peter Sieberer

L BI für A ngewandte K rebsforschung

K aiser-Franz-J osef-Spital

Kundratstraße 3, A -1100 Wien

Tel. +43 1 60191-2303, Fax -2329

E-mail christian.dittrich@kfj.magwien.gv.at

Auskuntt: Frau Dr. med. K. Liebold

Tagungsorganisation, $\mathrm{K}$ linik für $\mathrm{H}$ autkrankheiten und

A llergologie der Friedrich-Schiller-U niversität J ena

E rfurter Straße 35, D -07740 Jena

Tel. +49 3641 9373-22, Fax - 43

E-mail liebold@derma.uni-jena.de

..................................

Auskunft: Conference Secretariat «A B C-2001» Center for Tumor D etection and Prevention (ZeTuP)

R orschacher Straße 150, CH -9006 St. G allen

Tel. +41 71 24-30032, Fax -56805

E-mail eso-d@sg.zetup.ch

www.zetup.ch 


\section{02.-28. 02.2001}

München

D eutschland

\subsection{3.- 28. 03.2001}

New Orleans

USA

(...............

13.06.- 16.06.2001

Heidelberg

D eutschland

[.................

27.06.-30.06. 2001

Berlin

D eutschland

\section{.................}

18. 07.-21.07.2001

Zürich

Schweiz

[.................

Mannheim

D eutschland
Acute Leukemias IX - Basic Research, Experimental Approaches

American Association for Cancer Research - 92nd Annual Meeting

\section{ESPO 11. Psycho-Oncology in a United Europe - Changes \&} Challenges

\section{CARS 2001 - Computer Assisted Radiology and Surgery}

8th World Congress on Cancer of the Skin

Gemeinsame J ahrestagung der Deutschen und Österreichischen Gesellschaften für Hämatologie und Onkologie
Auskunft: Conference Secretariat «A BC-2001», Center for Tumor D etection and Prevention (ZeTuP) R orschacher Straße 150, CH -9006 St. G allen

Tel. +41 71 24-300 32, Fax -56805

E-mail eso-d@sg.zetup.ch

www.zetup.ch

Auskunft: A ACR

Public L edger Building, Suite 826

150 South Independence $M$ all West

Philadelphia, PA 19106-3483

Tel. +1215 440-9300, Fax -9313

www.aacr.org

Auskunft: Psychosoziale Nachsorgeeinrichtung

Chirurgische U niversitätsklinik

Frau Silke Ludwig

Im Neuenheimer Feld 155, D-69120 H eidelberg

Tel. +49 6221 56-2719, Fax -5250

E-mail silke_ludwig@med.uni-heidelberg.de

..............................

Auskunft: CA R S Conference 0 ffice

M rs. Franziska Schweikert

Im G ut 11/15, D-79790 Kuessaberg

Tel. +49 7742 91410, Fax -4391

E-mail francis.cars@d-plus.net

www.cars.tu-berlin.de

Auskunft: Dr. R einhard D ummer

$D$ ept. of D ermatology, $U$ niversity H ospital Zürich

G loriastraße 31, CH-8091 Zürich

Tel. +41 1 233-2507, Fax -4403

E-mail luethim@derm.unizh.ch

www.usz.unizh.ch/skincancer

\section{Auskunft: meet ideas}

R osengartenplatz 2, D-68161 M annheim

Tel. +49 621 4106-106, Fax -202

E-mail dgho.2001@meet-ideas.de

www.meet-ideas.de/dgho/

\title{
O GPO
}

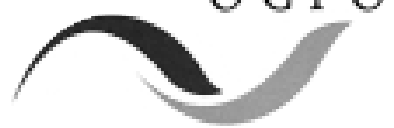

16. J ahrestagung der Österreichischen Gesellschaft für Psychoonkologie

\section{Zur Psychosomatik der Krebserkrankung - Zeitgemäße Therapeutische Ansätze}

\author{
Bad Ischl, 10. bis 12. November 2000
}

\section{Organisation: \\ E rika Wasserrab \\ $\mathrm{R}$ aiffeisenstraße 11 \\ A -2326 L anzendorf}

Tel. +43 22 35-472 30, Fax - 44816

E-mail erikawasserrab@yline.com 